\title{
SIGNED TOTAL ROMAN DOMINATION IN DIGRAPHS
}

\author{
Lutz Volkmann \\ Lehrstuhl II für Mathematik \\ RWTH Aachen University \\ 52056 Aachen, Germany \\ e-mail: volkm@math2.rwth-aachen.de
}

\begin{abstract}
Let $D$ be a finite and simple digraph with vertex set $V(D)$. A signed total Roman dominating function (STRDF) on a digraph $D$ is a function $f: V(D) \rightarrow\{-1,1,2\}$ satisfying the conditions that (i) $\sum_{x \in N^{-}(v)} f(x) \geq 1$ for each $v \in V(D)$, where $N^{-}(v)$ consists of all vertices of $D$ from which arcs go into $v$, and (ii) every vertex $u$ for which $f(u)=-1$ has an inner neighbor $v$ for which $f(v)=2$. The weight of an STRDF $f$ is $w(f)=$ $\sum_{v \in V(D)} f(v)$. The signed total Roman domination number $\gamma_{s t R}(D)$ of $D$ is the minimum weight of an STRDF on $D$. In this paper we initiate the study of the signed total Roman domination number of digraphs, and we present different bounds on $\gamma_{s t R}(D)$. In addition, we determine the signed total Roman domination number of some classes of digraphs. Some of our results are extensions of known properties of the signed total Roman domination number $\gamma_{s t R}(G)$ of graphs $G$.
\end{abstract}

Keywords: digraph, signed total Roman dominating function, signed total Roman domination number.

2010 Mathematics Subject Classification: 05C20, 05C69.

\section{REFERENCES}

[1] S. Arumugam, K. Jacop and L. Volkmann, Total and connected domination in digraphs, Australas. J. Combin. 39 (2007) 283-292.

[2] T.W. Haynes, S.T. Hedetniemi and P.J. Slater, Fundamentals of Domination in Graphs (Marcel Dekker, Inc., New York, 1998).

[3] T.W. Haynes, S.T. Hedetniemi and P.J. Slater, Editors, Domination in Graphs, Advanced Topics (Marcel Dekker, Inc., New York, 1998). 
[4] M.A. Henning, Signed total domination in graphs, Discrete Math. 278 (2004) 109-125. doi:10.1016/j.disc.2003.06.002

[5] E. Shan and T.C.E. Cheng, Remarks on the minus (signed) total domination in graphs, Discrete Math. 308 (2008) 3373-3380. doi:10.1016/j.disc.2007.06.015

[6] S.M. Sheikholeslami, Signed total domination numbers of directed graphs, Util. Math. 85 (2011) 213-218.

[7] S.M. Sheikholeslami and L. Volkmann, The Roman domination number of a digraph Acta Univ. Apulensis Math. Inform. 27 (2011) 77-96.

[8] L. Volkmann, Signed total Roman domination in graphs, J. Comb. Optim. 32 (2016) 855-871. doi 10.1007/s10878-015-9906-6

[9] B. Zelinka, Signed total domination numbers of a graph, Czechoslovak Math. J. 51 (2001) 225-229. doi:10.1023/A:1013782511179

Received 28 August 2015

Revised 15 April 2016

Accepted 15 April 2016 